\title{
Dietary Insulin Load and Cancer Recurrence and Survival in Patients With Stage III Colon Cancer: Findings From CALGB 89803 (Alliance)
}

\author{
Vicente Morales-Oyarvide, Chen Yuan, Ana Babic, Sui Zhang, Donna Niedzwiecki, Jennie C. \\ Brand-Miller, Laura Sampson-Kent, Xing Ye, Yanping Li, Leonard B. Saltz, Robert J. Mayer, \\ Rex B. Mowat, Renaud Whittom, Alexander Hantel, Al Benson, Daniel Atienza, Michael \\ Messino, Hedy Kindler, Alan Venook, Shuji Ogino, Kana Wu, Walter C. Willett, Edward L. \\ Giovannucci, Brian M. Wolpin, Jeffrey A. Meyerhardt, Charles S. Fuchs, Kimmie Ng
}

\begin{abstract}
Background: Evidence suggests that diets inducing postprandial hyperinsulinemia may be associated with increased cancerrelated mortality. The goal of this study was to assess the influence of postdiagnosis dietary insulin load and dietary insulin index on outcomes of stage III colon cancer patients.

Methods: We conducted a prospective observational study of 1023 patients with resected stage III colon cancer enrolled in an adjuvant chemotherapy trial who reported dietary intake halfway through and six months after chemotherapy. We evaluated the association of dietary insulin load and dietary insulin index with cancer recurrence and survival using Cox proportional hazards regression adjusted for potential confounders; statistical tests were two-sided.

Results: High dietary insulin load had a statistically significant association with worse disease-free survival (DFS), comparing the highest vs lowest quintile (adjusted hazard ratio $[\mathrm{HR}]=2.77,95 \%$ confidence interval $[\mathrm{CI}]=1.90$ to $4.02, P_{\text {trend }}<.001$ ). High dietary insulin index was also associated with worse DFS (highest vs lowest quintile, $\mathrm{HR}=1.75,95 \% \mathrm{CI}=1.22$ to $2.51, P_{\text {trend }}=$ .01). The association between higher dietary insulin load and worse DFS differed by body mass index and was strongest among patients with obesity $\left(\mathrm{HR}=3.66,95 \% \mathrm{CI}=1.88\right.$ to $\left.7.12, P_{\text {interaction }}=.04\right)$. The influence of dietary insulin load on cancer outcomes did not differ by mutation status of KRAS, BRAF, PIK3CA, TP53, or microsatellite instability.

Conclusions: Patients with resected stage III colon cancer who consumed a high-insulinogenic diet were at increased risk of recurrence and mortality. These findings support the importance of dietary management following resection of colon cancer, and future research into underlying mechanisms of action is warranted.
\end{abstract}

Postdiagnosis lifestyle behaviors, including diet and physical activity, may be important risk factors for cancer recurrence and death in colon cancer patients (1-3). The exact mechanisms through which these factors influence outcomes are unknown, but epidemiological evidence suggests that insulin may play a role $(4,5)$. Moreover, insulin signaling involves downstream pathways that are relevant for colon cancer pathogenesis and response to treatment (eg, KRAS/NRAS, BRAF, PIK3CA) $(6,7)$.

Insulin secretion is strongly influenced by diet, with carbohydrate-rich diets inducing postprandial hyperinsulinemia $(8,9)$. Dietary glycemic load-an indicator of the body's plasma glucose response to different foods-has been 
associated with adverse outcomes in patients with stage III colon cancer (10). Nevertheless, dietary glycemic load accounts for the insulinogenic response to dietary carbohydrates only, without considering the effects of protein- and fat-rich foods, which also stimulate insulin secretion and are known to act synergistically with carbohydrates $(9,11-13)$. The food insulin index (FII) is a novel parameter that quantifies the postprandial plasma insulin demand elicited by several foods, not just carbohydrates (11). Foods with higher FII evoke a stronger plasma insulin response; for instance, sweetened Greek yogurt has higher FII than bananas (86.3 vs 60.8 ), even though the carbohydrate content of Greek yogurt is much lower (11.6 grams vs 22.8 grams per 100-gram portion). Examples of foods with high FII include hard candies (FII = 120) and watermelon ( $F I I=95.3)$; in contrast, foods like raw almonds and green peppers have very low FII (5.6 and 3.5, respectively). Using the FII and dietary questionnaires, an individual's dietary insulin load and dietary insulin index, two dietary scores that predict the insulinogenic demand of the overall diet, can be calculated. These scores may shed light on the association between the body's insulin response to diet and survival outcomes in colon cancer survivors.

The goal of our study was to evaluate the association of dietary insulin load and dietary insulin index with disease recurrence and survival of patients with resected stage III colon cancer. We also explored whether microsatellite instability (MSI) and mutational status of KRAS, BRAF, PIK3CA, and TP53 modified the association between dietary insulin scores and patient outcomes.

\section{Methods}

\section{Study Population}

The patient population in this study was drawn from the National Cancer Institute-sponsored Cancer and Leukemia Group B (CALGB) phase III trial for patients with stage III colon cancer (CALGB 89803), comparing adjuvant chemotherapy with weekly 5-fluorouracil and leucovorin vs weekly irinotecan, 5fluorouracil, and leucovorin (ClinicalTrials.gov Identifier: NCT00003835) (14). CALGB is now part of the Alliance for Clinical Trials in Oncology. Between April 1999 and May 2001, 1264 patients were enrolled in the trial. Eligibility criteria included complete surgical resection of the primary tumor within 56 days before study enrollment and evidence of regional lymph node metastases without distant metastasis. Patients were also required to have adequate bone marrow, renal, and hepatic function, as well as a baseline Eastern Cooperative Oncology Group performance status of $0-2$. All patients signed informed consent, approved by each institution's review board.

A self-administered questionnaire of dietary and lifestyle habits was given to patients halfway through therapy (four months after surgery, questionnaire 1 [Q1]) and six months after completion of adjuvant therapy (14 months after surgery, questionnaire 2 [Q2]). The original trial protocol was amended to add dietary and lifestyle questionnaires after the first 87 patients were enrolled, leaving 1177 patients who were eligible. Figure 1 illustrates the derivation of the final study population of 1023 patients.

\section{Dietary Assessment}

Patients completed semiquantitative food frequency questionnaires (FFQ), developed and validated by Willett et al. $(15,16)$, that assessed 131 food items, vitamins, and mineral supplements. Participants answered questions regarding the average frequency with which they consumed a specific food portion size over the previous three months. Each question had up to nine possible answers, ranging from "never" to "six or more times per day." We calculated nutrient intakes by multiplying the frequency of consumption of each food by the nutrient content of the specified portions using composition values from Department of Agriculture sources supplemented with additional data (3). All nutrients were energy-adjusted using the residuals method (17).

\section{Exposure Assessment}

Dietary insulin load and dietary insulin index were calculated as a function of the FII of individual foods. We obtained the FII from previously published estimates $(11,18)$ or from Dr. Jennie Brand-Miller (University of Sydney, Sydney, Australia) and Laura Sampson-Kent (Harvard T. H. Chan School of Public Health, Boston, MA). Approximately 100 food items and 16 cereals were directly analyzed using a testing procedure previously described (11). Briefly, study subjects consumed a variety of isoenergetic $1000 \mathrm{~kJ}$ (240 kcal) foods and underwent blood insulin measurements every 15 minutes over 120 minutes. The FII was calculated by dividing the area under the insulin response curve (AUC) elicited by each test food by the AUC after ingesting 1000 $\mathrm{kJ}$ of the reference food (glucose). The FII scores for the remaining items included in the FFQ were recipe-derived, estimated using mathematical algorithms, or assumed to be zero. A complete list of the assessed food items and their FII scores can be obtained at the Harvard T. H. Chan School of Public Health Nutrition Department's file download site (https://regepi.bwh. harvard.edu/health/nutrition/index.html).

The dietary insulin load for each participant was calculated as follows: dietary insulin load $=(\Sigma[\mathrm{FII} \times$ kilocalories per serving $\times$ servings per day]). Each unit of dietary insulin load evokes an insulin response equivalent to that of $1-\mathrm{kcal}$ of glucose. The dietary insulin index for each participant's overall diet was computed using the following formula: dietary insulin index = ([dietary insulin load $\times 100] /$ total daily energy intake in kilocalories)). Given that dietary insulin load and dietary insulin index were measured at two time points (Q1 and Q2), we calculated a cumulative average for each dietary insulin score, weighted proportionally to the time elapsed between Q1 and Q2, and between Q2 and disease-free survival time, as described previously $(3,10,19)$. We also obtained weighted cumulative averages for additional dietary exposures (eg, "Western" and prudent dietary patterns [3], cereal fiber and alcohol intake), body mass index (BMI), and physical activity.

\section{Molecular Assessment for Microsatellite Instability and Mutations of KRAS, BRAF, TP53, and PIK3CA}

Polymerase chain reaction (PCR) of 10 microsatellite markers was used to assess MSI; if a tumor demonstrated instability in $50 \%$ or more loci, it was classified as MSI-high; for cases where PCR MSI results were not available $(n=28)$, those with MLH1 or MSH2 loss were classified as MSI-high (20). Targeted PCR and pyrosequencing were used to identify mutations in hotspots in KRAS codons 12 and 13 (21,22), BRAF codon 600 (23), and PIK3CA exons 9 and 20 (24), as previously described. Lastly, Sanger sequencing and sequencing by hybridization were used to evaluate mutations in TP53 exons 5-8 (25). 


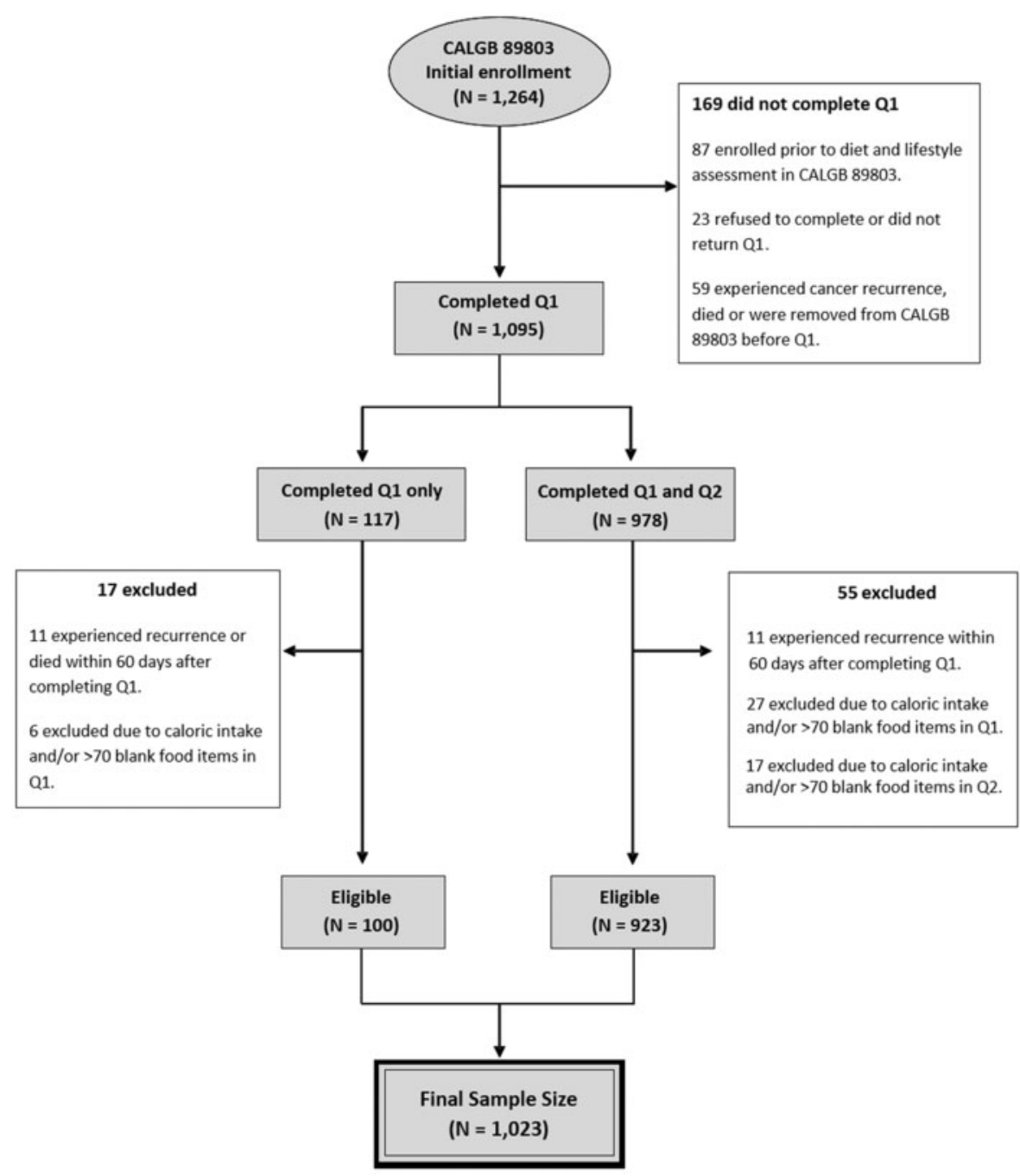

Figure 1. Patient flow diagram. Exclusions based on caloric intake: women, less than 500 kilocalories or greater than 3500 kilocalories per day; men, less than 600 kilocalories or greater than 4200 kilocalories per day. Q1 = questionnaire 1; Q2 = questionnaire 2.

\section{Study End Points}

The primary end point was disease-free survival (DFS), defined as time from completion of Q1 to colon cancer recurrence, occurrence of a new primary colon cancer, or death from any cause. Recurrence-free survival (RFS) was defined as time from completion of Q1 to colon cancer recurrence or occurrence of a new primary colon cancer, or death with evidence of recurrence; patients who died without evidence of recurrence were censored at last date of evaluation. Overall survival (OS) was defined as time from completion of Q1 to death from any cause. Follow-up occurred every three to four months for the first four years, then annually for three years, for a total of seven years of follow-up, and included serum carcinoembryonic antigen (CEA), chest x-rays, and computed tomography scans. First disease recurrence required biopsy confirmation.

\section{Statistical Analysis}

We first evaluated the correlation between the dietary insulin load and the dietary insulin index (as continuous variables) using the Pearson correlation coefficient. For survival outcomes analyses, the results of the main treatment trial showed no statistically significant differences in DFS or OS between the treatment arms (14); therefore, we combined patients from both arms of the trial and conducted statistical analyses based on quintiles of dietary insulin load and dietary insulin index. Survival end points were analyzed using the log-rank test and Kaplan-Meier curves (26). Multivariable-adjusted survival analyses evaluating the independent associations of the exposures of interest after adjusting for potential confounders were performed using Cox proportional hazards regression, calculating hazard ratios (HRs) and 95\% confidence intervals (CIs) (27). The proportionality of hazards assumption was tested by evaluating a time-dependent variable of the cross-product of the exposure and time, revealing a violation of the assumption for both dietary insulin scores $(P<.05$ each) (28). To address this issue, we included a term for the interaction between dietary insulin load or dietary insulin index and time in the Cox models, allowing for estimation of hazard ratios at different time points. Hazard ratios in the main analyses represent the average hazards of recurrence and death across the entire follow-up period; hazard ratios at one and three years after completion of Q1 are also presented.

Multivariable-adjusted analyses included the following covariates: sex (female, male), age (continuous), depth of 


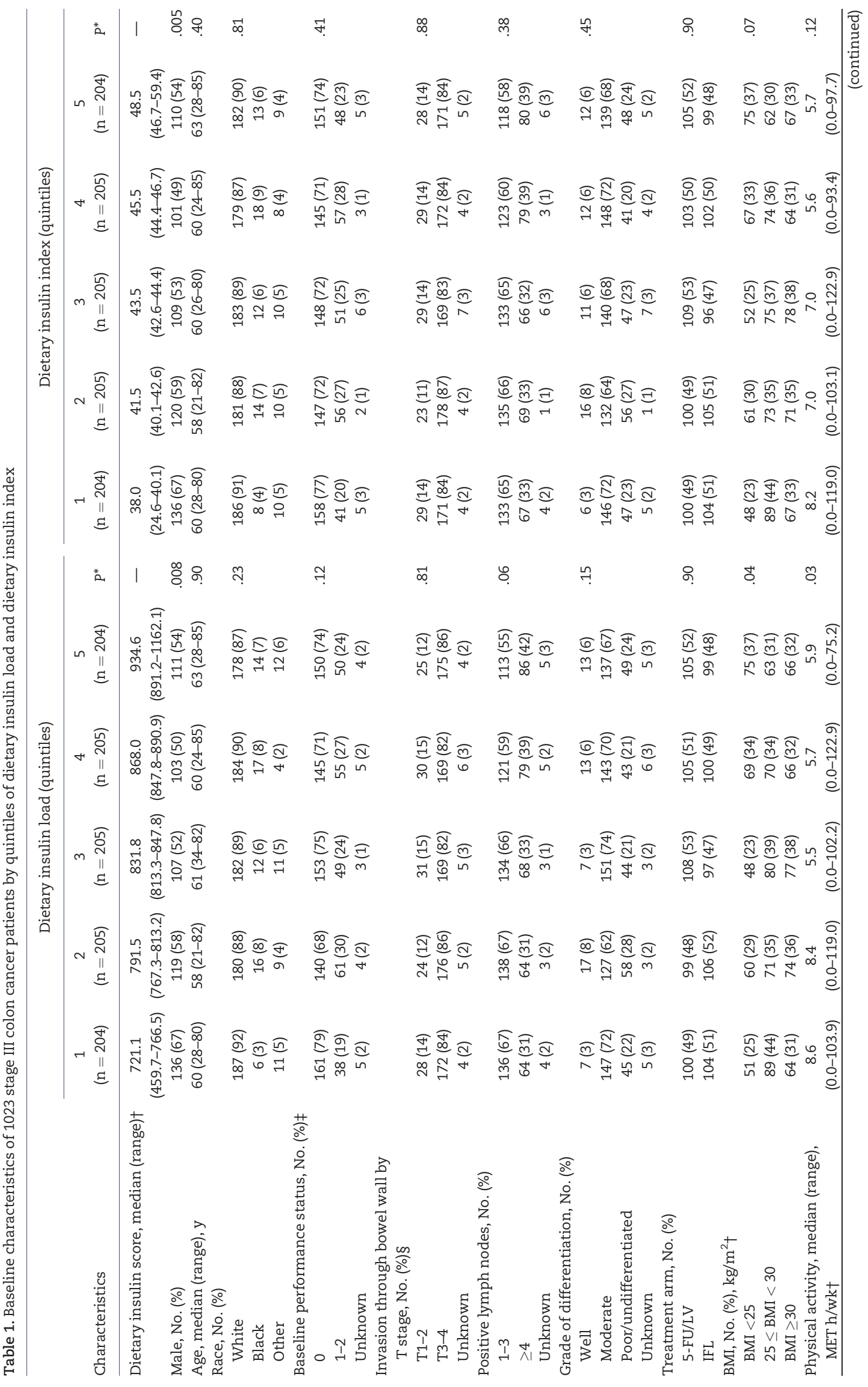


invasion through the bowel wall (T1-T2, T3-T4, unknown), number of positive lymph nodes (1-3, $\geq 4$, unknown), tumor grade (well-, moderately, poorly differentiated/undifferentiated, unknown), baseline performance status (0, 1-2, unknown), treatment group (5-FU/LV, IFL), body mass index (<25, 25-29.9, $\geq 30 \mathrm{~kg} / \mathrm{m}^{2}$ ), physical activity (continuous), cereal fiber (continuous), and alcohol intake (continuous). We conducted linear trend tests across quintiles of dietary insulin load and dietary insulin index by assigning each subject the median value for their corresponding quintile and modeling it as a continuous variable (1). We conducted tests of interactions by strata of potential effect modifiers by entering the cross-product of dietary insulin load (continuous) and the covariate of interest into the multivariable models and tested for statistical significance using the Wald test.

Statistical analyses were performed using SAS software (version 9.4; SAS Institute, Cary, NC). Statistical significance was set at a $P$ value of less than .05; all hypothesis tests were two-sided. Patient registration and clinical data collection were performed by the Alliance Statistics and Data Center. Statistical analyses were based on the study database frozen on November 9, 2009. Data quality was ensured by review of data by the Alliance Statistics and Data Center and by the study chairperson following Alliance policies.

\section{Results}

\section{Dietary Insulin Scores and Baseline Patient} Characteristics

The distribution of dietary insulin load and dietary insulin index in our study population is presented in Supplementary Figure 1 (available online); the scores were statistically correlated (Pearson correlation coefficient $=.98, P<.001$ ). The median dietary insulin load and dietary insulin index in our population (range) were 831.8 (459.7-1162.1) and 43.5 (24.6-59.4), respectively. Baseline characteristics of patients by quintiles of postdiagnosis dietary insulin scores are shown in Table 1. Patients with higher dietary insulin load and dietary insulin index were more likely to be female and to be less physically active, and to have higher dietary glycemic load, higher cereal fiber and total carbohydrate consumption, and lower alcohol intake.

\section{Dietary Insulin Load, Dietary Insulin Index, and Colon Cancer Recurrence and Mortality}

Median follow-up time among patients who were alive at the end of follow-up was 7.3 years. Higher dietary insulin load and dietary insulin index had statistically significant associations with worse DFS, RFS, and OS (Figure 2, Table 2). Patients with dietary insulin load in the highest quintile had an adjusted hazard ratio for DFS of 2.77 (95\% CI $=1.90$ to $\left.4.02, P_{\text {trend }}<.001\right)$, and this association persisted after further adjusting for dietary glycemic load (a list of food items that made a statistically significant contribution to elevated dietary insulin load independent of dietary glycemic load is presented in Supplementary Table 1, available online). We also found that the association of dietary insulin load with patient outcomes was independent of "Western" and prudent dietary patterns. Similarly, patients with dietary insulin index in the highest quintile had worse DFS compared with those in the lowest quintile (HR $=1.75,95 \% \mathrm{CI}=$ 1.22 to $\left.2.51, P_{\text {trend }}=.01\right)$. We obtained similar results in RFS and OS analyses (Table 2). Supplementary Tables 2 and 3 (available 

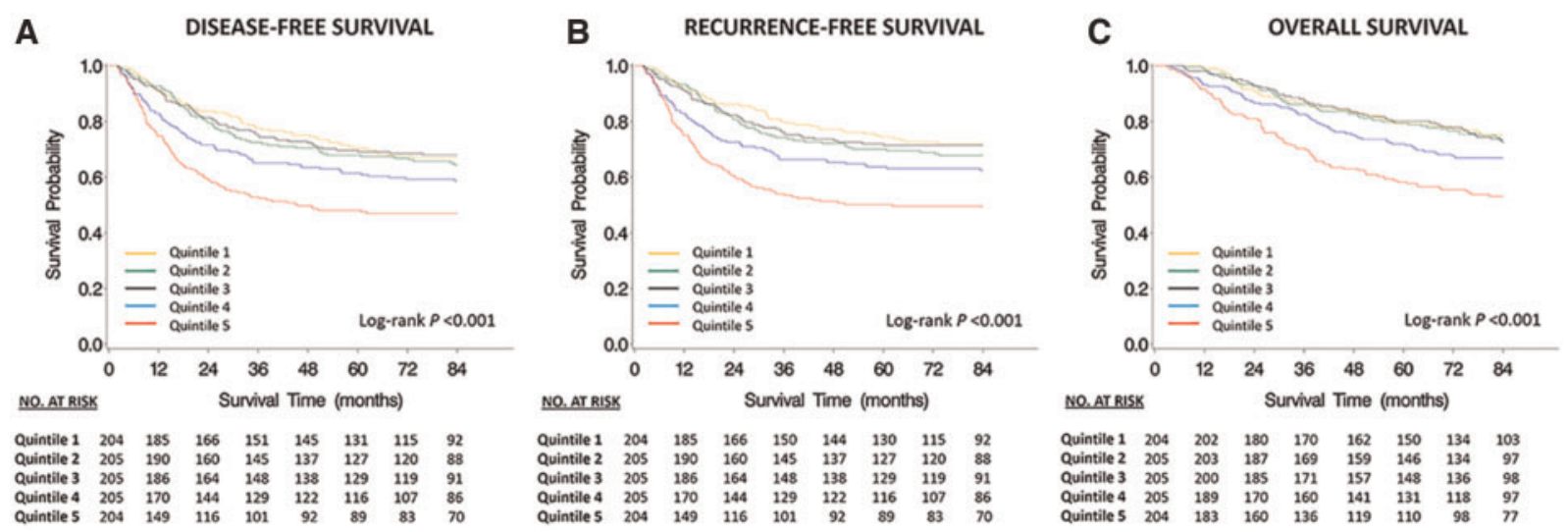

Figure 2. Kaplan-Meier survival curves by quintiles of dietary insulin load. A) Disease-free survival. B) Recurrence-free survival. C) Overall survival. All statistical tests were two-sided.

Table 2. Relationship between dietary insulin load*, dietary insulin index*, and colon cancer recurrence and mortality

\begin{tabular}{|c|c|c|c|c|c|c|}
\hline \multirow[b]{2}{*}{ Dietary insulin scores and survival outcomes } & \multicolumn{5}{|c|}{ Quintiles } & \multirow[b]{2}{*}{$P_{\text {trend }} \dagger$} \\
\hline & $\begin{array}{c}1 \\
(\mathrm{n}=204)\end{array}$ & $\begin{array}{c}2 \\
(\mathrm{n}=205)\end{array}$ & $\begin{array}{c}3 \\
(\mathrm{n}=205)\end{array}$ & $\begin{array}{c}4 \\
(\mathrm{n}=205)\end{array}$ & $\begin{array}{c}5 \\
(\mathrm{n}=204)\end{array}$ & \\
\hline \multicolumn{7}{|l|}{ Dietary insulin load } \\
\hline \multicolumn{7}{|l|}{ Disease-free survival } \\
\hline No. of events & 67 & 72 & 66 & 86 & 107 & \\
\hline Age-adjusted only, HR (95\% CI) & 1 (referent) & 1.10 (0.79 to 1.53$)$ & 1.00 (0.71 to 1.40$)$ & 1.42 (1.03 to 1.96$)$ & 2.08 (1.54 to 2.83$)$ & $<.001$ \\
\hline Multivariable-adjusted, HR $(95 \%$ CI)‡ & 1 (referent) & 1.35 (0.93 to 1.96$)$ & $1.30(0.89$ to 1.91$)$ & 1.90 (1.30 to 2.77$)$ & 2.77 (1.90 to 4.02$)$ & $<.001$ \\
\hline Multivariable-adjusted, HR (95\% CI)§ & 1 (referent) & 1.23 (0.84 to 1.81$)$ & 1.13 (0.74 to 1.70$)$ & 1.59 (1.05 to 2.42$)$ & 2.13 (1.34 to 3.40$)$ & $<.001$ \\
\hline \multicolumn{7}{|l|}{ Recurrence-free survival } \\
\hline No. of events & 54 & 65 & 57 & 78 & 100 & \\
\hline Age-adjusted only, HR (95\% CI) & 1 (referent) & $1.22(0.85$ to 1.75$)$ & 1.07 (0.74 to 1.55$)$ & 1.61 (1.14 to 2.27$)$ & 2.39 (1.72 to 3.33$)$ & $<.001$ \\
\hline Multivariable-adjusted, HR (95\% CI)‡ & 1 (referent) & 1.47 (0.98 to 2.20$)$ & 1.36 (0.89 to 2.07$)$ & 2.09 (1.39 to 3.14$)$ & 3.07 (2.05 to 4.60$)$ & $<.001$ \\
\hline Multivariable-adjusted, HR (95\% CI)§ & 1 (referent) & 1.34 (0.88 to 2.02$)$ & 1.17 (0.75 to 1.83$)$ & 1.74 (1.11 to 2.74$)$ & 2.35 (1.43 to 3.86$)$ & $<.001$ \\
\hline \multicolumn{7}{|l|}{ Overall survival } \\
\hline No. of events & 53 & 51 & 56 & 64 & 95 & \\
\hline Age-adjusted only, HR (95\% CI) & 1 (referent) & 0.97 (0.66 to 1.43$)$ & 1.04 (0.72 to 1.52$)$ & 1.30 (0.90 to 1.87$)$ & 2.21 (1.58 to 3.09$)$ & $<.001$ \\
\hline Multivariable-adjusted, HR (95\% CI)キ & 1 (referent) & 1.18 (0.77 to 1.83$)$ & 1.38 (0.90 to 2.12$)$ & 1.75 (1.14 to 2.70$)$ & 2.98 (1.96 to 4.51$)$ & $<.001$ \\
\hline Multivariable-adjusted, HR (95\% CI)§ & 1 (referent) & 1.09 (0.70 to 1.69$)$ & 1.20 (0.75 to 1.90$)$ & 1.48 (0.92 to 2.38$)$ & 2.30 (1.36 to 3.87$)$ & $<.001$ \\
\hline \multicolumn{7}{|l|}{ Dietary insulin index } \\
\hline \multicolumn{7}{|l|}{ Disease-free survival } \\
\hline No. of events & 73 & 83 & 76 & 72 & 94 & \\
\hline Age-adjusted only, HR (95\% CI) & 1 (referent) & 1.20 (0.88 to 1.64$)$ & 1.08 (0.78 to 1.49$)$ & 0.99 (0.72 to 1.37$)$ & 1.48 (1.09 to 2.00$)$ & .06 \\
\hline Multivariable-adjusted, HR (95\% CI)‡ & 1 (referent) & 1.38 (0.97 to 1.95$)$ & $1.26(0.88$ to 1.81$)$ & 1.16 (0.80 to 1.69$)$ & 1.75 (1.22 to 2.51$)$ & .01 \\
\hline Multivariable-adjusted, HR (95\% CI)\| & 1 (referent) & 1.39 (0.98 to 1.98$)$ & 1.28 (0.88 to 1.98$)$ & $1.18(0.80$ to 1.74$)$ & 1.78 (1.21 to 2.64$)$ & .02 \\
\hline \multicolumn{7}{|l|}{ Recurrence-free survival } \\
\hline No. of events & 60 & 76 & 70 & 60 & 88 & \\
\hline Age-adjusted only, HR (95\% CI) & 1 (referent) & 1.32 (0.94 to 1.85$)$ & 1.21 (0.86 to 1.71$)$ & 1.00 (0.70 to 1.43$)$ & 1.68 (1.21 to 2.34$)$ & .02 \\
\hline Multivariable-adjusted, HR (95\% CI)‡ & 1 (referent) & 1.48 (1.02 to 2.14$)$ & 1.36 (0.93 to 1.99$)$ & 1.13 (0.75 to 1.69$)$ & 1.90 (1.29 to 2.79$)$ & .009 \\
\hline Multivariable-adjusted, HR (95\% CI)\| & 1 (referent) & 1.48 (1.01 to 2.15$)$ & $1.36(0.91$ to 2.01$)$ & $1.12(0.74$ to 1.72$)$ & 1.89 (1.25 to 2.87$)$ & .02 \\
\hline \multicolumn{7}{|l|}{ Overall survival } \\
\hline No. of events & 58 & 63 & 61 & 56 & 81 & \\
\hline Age-adjusted only, HR (95\% CI) & 1 (referent) & 1.14 (0.80 to 1.63$)$ & 1.05 (0.80 to 1.63$)$ & 0.97 (0.67 to 1.40$)$ & 1.53 (1.09 to 2.14$)$ & .04 \\
\hline Multivariable-adjusted, HR (95\% CI) & 1 (referent) & 1.28 (0.86 to 1.90$)$ & 1.26 (0.84 to 1.88$)$ & 1.11 (0.73 to 1.70$)$ & 1.84 (1.23 to 2.76$)$ & .007 \\
\hline Multivariable-adjusted, HR (95\% CI)\| & 1 (referent) & 1.29 (0.87 to 1.93$)$ & 1.27 (0.84 to 1.93$)$ & $1.13(0.73$ to 1.76$)$ & 1.89 (1.22 to 2.91$)$ & .009 \\
\hline
\end{tabular}

*Using cumulative averaging from questionnaire 1 and questionnaire $2 . \mathrm{CI}=$ confidence interval; $\mathrm{HR}=$ hazard ratio.

†Two-sided Wald test.

$\ddagger$ Multivariable-adjusted model adjusted for sex (female, male), age (continuous variable), depth of invasion through bowel wall (T1-T2, T3-T4, unknown), number of positive lymph nodes (1-3, $\geq 4$, unknown), tumor grade (well-, moderately, poorly differentiated/undifferentiated, unknown), baseline performance status (0, 1-2, unknown), treatment group (5-FU/LV, IFL), body mass index $\left(<25,25-29.9, \geq 30 \mathrm{~kg} / \mathrm{m}^{2}\right)$, physical activity (continuous variable), cereal fiber (continuous variable), and alcohol intake (continuous variable).

§Additionally adjusted for glycemic load (continuous variable).

|Additionally adjusted for glycemic index (continuous variable). 
Table 3. Disease-free survival by quintiles of dietary insulin load stratified by clinicopathological features*

\begin{tabular}{|c|c|c|c|c|c|c|c|}
\hline \multirow[b]{2}{*}{ Subgroup } & \multirow[b]{2}{*}{$\begin{array}{c}\text { No. of } \\
\text { patients }\end{array}$} & \multicolumn{5}{|c|}{ Quintiles of dietary insulin load } & \multirow[b]{2}{*}{$P_{\text {interaction }}$} \\
\hline & & $\begin{array}{c}1 \\
(\mathrm{n}=204) \\
\text { HR }(95 \% \mathrm{CI})\end{array}$ & $\begin{array}{c}2 \\
(\mathrm{n}=205) \\
\mathrm{HR}(95 \% \mathrm{CI})\end{array}$ & $\begin{array}{c}3 \\
(\mathrm{n}=205) \\
\text { HR }(95 \% \mathrm{CI})\end{array}$ & $\begin{array}{c}4 \\
(\mathrm{n}=205) \\
\mathrm{HR}(95 \% \mathrm{CI})\end{array}$ & $\begin{array}{c}5 \\
(\mathrm{n}=204) \\
\mathrm{HR}(95 \% \mathrm{CI})\end{array}$ & \\
\hline \multicolumn{8}{|l|}{ Age, y } \\
\hline$<60$ & 488 & 1 (referent) & 1.39 (0.82 to 2.35$)$ & 1.19 (0.66 to 2.12 ) & 2.07 (1.20 to 3.57$)$ & 2.33 (1.34 to 4.04$)$ & .89 \\
\hline$\geq 60$ & 535 & 1 (referent) & 1.33 (0.78 to 2.28$)$ & 1.47 (0.87 to 2.48$)$ & 1.88 (1.10 to 3.19$)$ & 3.25 (1.93 to 5.46$)$ & \\
\hline \multicolumn{8}{|l|}{ Sex } \\
\hline Male & 576 & 1 (referent) & 1.20 (0.77 to 1.89$)$ & 1.11 (0.69 to 1.78$)$ & 1.51 (0.94 to 2.44$)$ & 2.64 (1.68 to 4.16$)$ & .53 \\
\hline Female & 447 & 1 (referent) & 1.90 (0.94 to 3.79$)$ & 1.85 (0.90 to 3.79 ) & 2.90 (1.43 to 5.87$)$ & 3.50 (1.71 to 7.16$)$ & \\
\hline \multicolumn{8}{|c|}{ Baseline performance status $\ddagger$} \\
\hline 0 & 749 & 1 (referent) & 1.57 (1.00 to 2.45 ) & 1.27 (0.80 to 2.03 ) & 2.21 (1.40 to 3.48 ) & 2.98 (1.91 to 4.65$)$ & .81 \\
\hline $1-2$ & 253 & 1 (referent) & 0.94 (0.46 to 1.94$)$ & 1.20 (0.56 to 2.55$)$ & 1.39 (0.66 to 2.92$)$ & 2.23 (1.05 to 4.75$)$ & \\
\hline \multicolumn{8}{|c|}{ No. of positive lymph nodes } \\
\hline 1-3 (N1) & 642 & 1 (referent) & 1.20 (0.75 to 1.93$)$ & 1.19 (0.72 to 1.96$)$ & 1.76 (1.08 to 2.87 ) & 2.70 (1.66 to 4.39$)$ & .89 \\
\hline$\geq 4(\mathrm{~N} 2)$ & 361 & 1 (referent) & $1.96(1.02$ to 3.75$)$ & 1.55 (0.81 to 2.99$)$ & 2.45 (1.29 to 4.64$)$ & 3.19 (1.69 to 6.04$)$ & \\
\hline \multicolumn{8}{|c|}{ 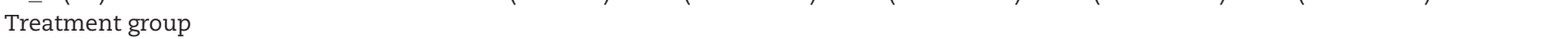 } \\
\hline 5-FU/LV & 517 & 1 (referent) & 1.19 (0.70 to 2.02 ) & 1.12 (0.65 to 1.95$)$ & 1.55 (0.90 to 2.67 ) & 2.17 (1.27 to 3.71$)$ & .10 \\
\hline IFL & 506 & 1 (referent) & $1.46(0.85$ to 2.51$)$ & 1.54 (0.88 to 2.67$)$ & 2.42 (1.42 to 4.14$)$ & 3.54 (2.07 to 6.05$)$ & \\
\hline \multicolumn{8}{|c|}{ - The } \\
\hline $\mathrm{BMI}<25$ & 303 & 1 (referent) & 1.11 (0.57 to 2.17$)$ & 0.99 (0.47 to 2.08$)$ & 1.66 (0.83 to 3.29$)$ & 2.02 (1.02 to 3.99$)$ & .04 \\
\hline $25 \leq \mathrm{BMI}<30$ & 373 & 1 (referent) & 1.37 (0.69 to 2.73$)$ & 1.46 (0.76 to 2.81$)$ & 1.85 (0.96 to 3.56$)$ & 3.08 (1.61 to 5.89$)$ & \\
\hline $\mathrm{BMI} \geq 30$ & 347 & 1 (referent) & 1.62 (0.87 to 3.11$)$ & 1.56 (0.80 to 3.05$)$ & 2.43 (1.23 to 4.77$)$ & 3.66 (1.88 to 7.12$)$ & \\
\hline \multicolumn{8}{|c|}{ Physical activity, MET-hours/wk } \\
\hline$<18$ & 775 & 1 (referent) & 1.22 (0.79 to 1.89$)$ & 1.23 (0.80 to 1.89$)$ & 1.52 (0.98 to 2.36$)$ & 2.69 (1.76 to 4.10$)$ & .46 \\
\hline$\geq 18$ & 248 & 1 (referent) & 1.97 (0.95 to 4.09$)$ & 1.35 (0.53 to 3.48$)$ & 3.67 (1.66 to 8.10$)$ & 2.52 (1.05 to 6.06$)$ & \\
\hline \multicolumn{8}{|c|}{ Dietary glycemic load } \\
\hline$\leq 142$ & 511 & 1 (referent) & $1.28(0.84$ to 1.95$)$ & 1.12 (0.68 to 1.84$)$ & 1.70 (0.99 to 2.94$)$ & 2.34 (1.10 to 4.95$)$ & .40 \\
\hline$>142$ & 512 & 1 (referent) & 0.79 (0.11 to 1.98$)$ & 0.82 (0.34 to 1.97$)$ & $1.19(0.50$ to 2.81$)$ & 1.71 (0.72 to 4.04$)$ & \\
\hline
\end{tabular}

"Multivariable-adjusted model adjusted for sex (female, male), age (continuous variable), depth of invasion through bowel wall (T1-T2, T3-T4, unknown), number of positive lymph nodes (1-3, $\geq 4$, unknown), tumor grade (well-, moderately, poorly differentiated/undifferentiated, unknown), baseline performance status (0, 1-2, unknown), treatment group (5-FU/LV, IFL), body mass index $\left(<25,25-29.9, \geq 30 \mathrm{~kg} / \mathrm{m}^{2}\right)$, physical activity (continuous variable), cereal fiber (continuous variable), and alcohol intake (continuous variable). 5-FU = 5-fluorouracil; BMI = body mass index; $\mathrm{CI}$ = confidence interval; $\mathrm{HR}$ = hazard ratio; IFL = irinotecan, 5-fluorouracil, leucovorin; $\mathrm{LV}=$ leucovorin; $\mathrm{MET}=$ metabolic equivalent tasks.

†Interaction term built as a cross-product of dietary insulin load as a continuous variable, and the covariate of interest as a categorical variable. All statistical tests were two-sided.

$\ddagger$ Baseline performance status: Performance status 0 = fully active; Performance status 1 = restricted in physically strenuous activity but ambulatory and able to carry out light work; Performance status 2 = ambulatory and capable of all self-care but unable to carry out any work activities, up and about more than $50 \%$ of waking hours.

online) show the hazard ratio estimates corresponding to one and three years after completion of Q1. In sensitivity analyses modeling dietary insulin scores as time-dependent variables, the statistically significant associations between higher dietary insulin load and dietary insulin index and worse DFS $\left(P_{\text {trend }}<\right.$ .001 and $P_{\text {trend }}=.008$, respectively) persisted.

\section{Subgroup Analyses}

We evaluated the influence of dietary insulin load on disease recurrence and mortality across strata of potential effectmodifying variables. The association between dietary insulin load and DFS was statistically modified by BMI $\left(P_{\text {interaction }}=.04\right)$, with the magnitude of the association being highest for patients with obesity (Table 3). Dietary insulin load in the highest quintile had a statistically significant association with worse DFS in all BMI categories (patients with BMI $<25 \mathrm{~kg} / \mathrm{m}^{2}$ : $\mathrm{HR}=2.02,95 \%$ $\mathrm{CI}=1.02$ to 3.99 ; overweight patients $\left[25 \mathrm{~kg} / \mathrm{m}^{2} \leq \mathrm{BMI}<30 \mathrm{~kg}\right.$ / $\mathrm{m}^{2}$ ]: $\mathrm{HR}=3.08,95 \% \mathrm{CI}=1.61$ to 5.89 ; obese patients $[\mathrm{BMI} \geq 30$ $\left.\mathrm{kg} / \mathrm{m}^{2}\right]: \mathrm{HR}=3.66,95 \% \mathrm{CI}=1.88$ to $\left.7.12 ; P_{\text {interaction }}=.04\right)$. There was no evidence of statistically significant interactions with other clinical characteristics or tumor genomic profiles (Tables 3 and 4).

\section{Discussion}

In a population of resected stage III colon cancer patients enrolled in an adjuvant therapy trial, higher postdiagnosis dietary insulin load and dietary insulin index were associated with increased risk of disease recurrence and mortality. The associations persisted after adjusting for dietary glycemic load and dietary glycemic index, suggesting that protein- and fat-rich foods elicit a postprandial insulin response that may influence cancer outcomes beyond dietary carbohydrates. The association of dietary insulin scores with patient outcomes was independent of "Western" and prudent dietary patterns; however, these results must be interpreted cautiously given that several of the same food items are determinants of dietary insulin scores, as well as of the "Western" and prudent patterns (3), raising concerns about collinearity. Although we can hypothesize about the individual foods that contribute to high dietary insulin scores, the dietary insulin scores were designed to predict the 
Table 4. Disease-free survival by tertiles of dietary insulin load stratified by molecular status*

\begin{tabular}{|c|c|c|c|c|c|}
\hline \multirow[b]{2}{*}{ Subgroup } & \multirow[b]{2}{*}{ No. of patients } & \multicolumn{3}{|c|}{ Tertiles of dietary insulin load } & \multirow[b]{2}{*}{$P_{\text {interaction }} \dagger$} \\
\hline & & $\begin{array}{c}1 \\
(\mathrm{n}=341) \\
\mathrm{HR}(95 \% \mathrm{CI})\end{array}$ & $\begin{array}{c}2 \\
(\mathrm{n}=341) \\
\mathrm{HR}(95 \% \mathrm{CI})\end{array}$ & $\begin{array}{c}3 \\
(\mathrm{n}=341) \\
\mathrm{HR}(95 \% \mathrm{CI})\end{array}$ & \\
\hline \multicolumn{6}{|l|}{ KRAS } \\
\hline Wild-type & 360 & 1 (referent) & 1.70 (1.03 to 2.80$)$ & 3.21 (1.92 to 5.37$)$ & .27 \\
\hline Mutant & 183 & 1 (referent) & 1.41 (0.67 to 2.97$)$ & 2.46 (1.17 to 5.17$)$ & \\
\hline \multicolumn{6}{|l|}{ BRAF } \\
\hline Wild-type & 461 & 1 (referent) & 1.43 (0.93 to 2.20 ) & 2.54 (1.64 to 3.95$)$ & .16 \\
\hline Mutant & 81 & 1 (referent) & $2.62(0.72$ to 9.51$)$ & 8.57 (2.28 to 32.45$)$ & \\
\hline \multicolumn{6}{|l|}{ PIK3CA } \\
\hline Wild-type & 456 & 1 (referent) & 1.53 (0.98 to 2.38$)$ & $2.70(1.74$ to 4.20$)$ & .93 \\
\hline Mutant & 61 & 1 (referent) & $3.70(0.72$ to 19.11$)$ & 6.04 (0.82 to 44.50$)$ & \\
\hline \multicolumn{6}{|l|}{ TP53 } \\
\hline Wild-type & 247 & 1 (referent) & 2.64 (1.42 to 4.93$)$ & 2.29 (1.19 to 4.42$)$ & .71 \\
\hline Mutant & 211 & 1 (referent) & $0.68(0.37$ to 1.27$)$ & 2.57 (1.46 to 4.53$)$ & \\
\hline \multicolumn{6}{|c|}{ Microsatellite instability } \\
\hline Low & 904 & 1 (referent) & $1.38(1.02$ to 1.86$)$ & 2.27 (1.69 to 3.05$)$ & .88 \\
\hline High & 119 & 1 (referent) & 3.18 (1.05 to 9.60$)$ & 6.48 (1.94 to 21.58$)$ & \\
\hline
\end{tabular}

${ }^{*}$ Multivariable-adjusted model adjusted for sex (female, male), age (continuous variable), depth of invasion through bowel wall (T1-T2, T3-T4, unknown), number of positive lymph nodes (1-3, $\geq 4$, unknown), tumor grade (well-, moderately, poorly differentiated/undifferentiated, unknown), baseline performance status (0, 1-2, unknown), treatment group (5-FU/LV, IFL), body mass index $\left(<25,25-29.9, \geq 30 \mathrm{~kg} / \mathrm{m}^{2}\right)$, physical activity (continuous variable), cereal fiber (continuous variable), and alcohol intake (continuous variable). $\mathrm{CI}=$ confidence interval; $\mathrm{HR}=$ hazard ratio.

†Interaction term built as a cross-product of dietary insulin load as a continuous variable, and the covariate of interest as a binary variable. All statistical tests were two-sided.

insulinogenic effect of the overall diet rather than any particular food in isolation.

The magnitude of the association with disease recurrence and survival was higher for dietary insulin load than it was for dietary insulin index, suggesting that food energy content plays an important role in this association. Moreover, the association was stronger at one year after initial dietary assessment than at three years. This attenuation could be due to several reasons, including an increase in non-cancer-related deaths as time passes from colon cancer diagnosis, different biological factors influencing early vs late disease recurrence, and changes in dietary patterns over time.

The influence of dietary insulin load on outcomes was strongest among patients who were overweight or obese. Obesity is associated with insulin resistance, which is accompanied by compensatory hyperinsulinemia $(29,30)$. Overweight and obese patients in our series might have had elevated basal levels of circulating insulin that were further increased by high dietary insulin load, resulting in higher plasma insulin than a lean patient following the same diet. We recently reported similar findings in patients from two large prospective cohorts in the United States diagnosed with all-stage colorectal cancer (CRC) (31). Consistent with our results, high dietary insulin load and index were associated with high CRC-specific and overall mortality, and the association was more evident among patients who were overweight or obese. Collectively, our results indicate that a diet that strongly stimulates postprandial insulin secretion is associated with worse clinical outcomes, independent of BMI and physical activity. This finding is consistent with our previous study that reported that elevated circulating C-peptide is associated with higher mortality in CRC patients (5).

Experimental studies have shown that insulin activates mitogenic and antiapoptotic pathways in tumor cells $(32,33)$. Activation of the insulin receptor leads to PI3-kinase(PI3K)/Akt pathway signaling, which modulates genes involved in cell cycle regulation and apoptosis (6); it also triggers the Ras-MAPK pathway, which involves the activation of Ras-Raf-MEK1/2ERK1/2 and promotes cell proliferation (6). Several cancer types-including CRC-express insulin receptors, and their activation can lead to increased cell proliferation, inhibition of apoptosis, and metastasis (32-34). We did not find statistically significant differences in the influence of insulin load on survival based on KRAS, BRAF, PIK3CA, and TP53 mutation status or microsatellite instability, possibly due to lack of statistical power or to mechanisms beyond those captured by these molecular factors.

The present study has several strengths. The FII of the food items assessed by the FFQ has been validated as an adequate representation of the plasma insulin response elicited by those specific foods $(9,11)$. Further, the FII has also been shown to predict the postprandial insulin response to realistic mixed meals, which is of great relevance given the synergistic effect of proteins and carbohydrates to stimulate insulin secretion (9) and the importance of overall dietary patterns (3). Our study was conducted in a population derived from a randomized controlled trial with highly annotated clinical and molecular information, which offers several advantages over traditional observational studies: the baseline clinical characteristics are similar across participants; treatment regimens were highly controlled; follow-up was standardized to streamline ascertainment of disease recurrence and cause of death; and molecular data allowed us to explore interactions between dietary insulin scores and tumor genomic status. Dietary and lifestyle factors were assessed during and six months after completion of adjuvant chemotherapy, allowing for evaluation of changes in dietary habits over time. Although diet and physical activity were self-reported, the questionnaire used in this study has been validated in both healthy populations and cancer patients undergoing chemotherapy $(16,35)$, as well as in studies of cardiovascular disease $(36,37)$ and overall mortality $(38,39)$. 
Our study also has some limitations. Participants in randomized treatment trials need to satisfy stringent criteria to enroll and be motivated to be part of the study, which may raise concerns about generalizability. However, CALGB 89803 enrolled patients from both community and academic centers across North America, and we have not observed statistically significant dietary differences between our study population and that of the general population (10). Second, if patients in our study had similar pre- and postdiagnosis dietary habits, it is plausible that patients with low prediagnosis dietary insulin load and dietary insulin index had biologically different tumors with more indolent behavior. Nevertheless, this is unlikely because we did not observe statistically significant molecular interactions between dietary insulin load and patient survival. Our main outcome analyses modeled dietary insulin scores as fixed time-independent variables obtained by cumulative averaging, introducing potential for bias; however, sensitivity analyses modeling the scores as time-dependent variables did not result in statistically significant changes to our results.

In summary, we show that postdiagnosis dietary insulin load and dietary insulin index adversely influence patient outcomes in resected stage III colon cancer. Our findings strongly suggest that dietary factors play a role in colon cancer recurrence and survival following resection. Future research efforts should be directed toward understanding the underlying mechanisms of action and impact of dietary counseling on patient survival.

\section{Funding}

This work was supported by the National Cancer Institute of the National Institutes of Health (U10CA032291, U10CA041287, U10CA045808, U10CA077651, U10CA138561, U10CA180791, U10CA180836, and U10CA180867; U10CA180821 and U10CA180882 to the Alliance for Clinical Trials in Oncology; R35CA197735 to SO; R01CA118553, R01CA169141, and P50CA127003 to CSF; R01CA149222 to JAM; K07CA148894 and R01CA205406 to KN); the Project P Fund to CSF; and Stand Up to Cancer Colorectal Cancer Dream Team Grant to CSF.

\section{Notes}

Affiliations of authors: Department of Medical Oncology, DanaFarber Cancer Institute, Boston, MA (VMO, CY, AB, SZ, RJM, SO, BMW, JAM, KN); Alliance Statistics and Data Center, Duke University, Durham, NC (DN, XY); Institute of Obesity, Nutrition, and Exercise, University of Sydney, Sydney, Australia (JCBM); Department of Nutrition (LSK, YL, KW, WCW) and Department of Epidemiology (SO, WCW, ELG), Harvard T. H. Chan School of Public Health, Boston, MA; Memorial Sloan Kettering Cancer Center, New York, NY (LBS); Toledo Community Hospital Oncology Program, Toledo, OH (RBM); Hôpital du Sacré-Coeur de Montréal, Montreal, Canada (RW); Loyola University Stritch School of Medicine, Naperville, IL (AH); Robert H. Lurie Comprehensive Cancer Center, Northwestern University, Chicago, IL (AB); Virginia Oncology Associates, Norfolk, VA (DA); Southeast Clinical Oncology Research Consortium, Mission Hospitals, Asheville, NC (MM); University of Chicago Comprehensive Cancer Center, Chicago, IL (HK); University of California at San Francisco Comprehensive Cancer Center, San Francisco, CA (AV); Department of Pathology, Brigham and
Women's Hospital, Boston, MA (SO); Department of Pathology, Harvard Medical School, Boston, MA (SO); Channing Laboratory, Department of Medicine, Brigham and Women's Hospital and Harvard Medical School, Boston, MA (ELG); Yale Cancer Center, Smilow Cancer Hospital and Yale School of Medicine, New Haven, CT (CSF).

The funders had no role in the design of the study; the collection, analysis, or interpretation of the data; the writing of the manuscript; or the decision to submit the manuscript for publication.

The authors have no conflicts of interest to declare.

\section{References}

1. Meyerhardt JA, Heseltine D, Niedzwiecki D, et al. Impact of physical activity on cancer recurrence and survival in patients with stage III colon cancer: Findings from CALGB 89803. J Clin Oncol. 2006;24(22):3535-3541.

2. Meyerhardt JA, Giovannucci EL, Holmes MD, et al. Physical activity and survival after colorectal cancer diagnosis. J Clin Oncol. 2006;24(22):3527-3534.

3. Meyerhardt JA, Niedzwiecki D, Hollis D, et al. Association of dietary patterns with cancer recurrence and survival in patients with stage III colon cancer. JAMA. 2007;298(7):754-764.

4. Mills KT, Bellows CF, Hoffman AE, et al. Diabetes mellitus and colorectal cancer prognosis: A meta-analysis. Dis Colon Rectum. 2013;56(11):1304-1319.

5. Wolpin BM, Meyerhardt JA, Chan AT, et al. Insulin, the insulin-like growth factor axis, and mortality in patients with nonmetastatic colorectal cancer. J Clin Oncol. 2009;27(2):176-185.

6. Boucher J, Kleinridders A, Kahn CR. Insulin receptor signaling in normal and insulin-resistant states. Cold Spring Harb Perspect Biol. 2014;6(1).

7. Zenonos K, Kyprianou K. RAS signaling pathways, mutations and their role in colorectal cancer. World J Gastrointest Oncol. 2013;5(5):97-101.

8. Garg A, Bantle JP, Henry RR, et al. Effects of varying carbohydrate content of diet in patients with non-insulin-dependent diabetes mellitus. JAMA. 1994; 271(18):1421-1418.

9. Bao J, de Jong V, Atkinson F, et al. Food insulin index: Physiologic basis for predicting insulin demand evoked by composite meals. Am J Clin Nutr. 2009; 90(4):986-992.

10. Meyerhardt JA, Sato K, Niedzwiecki D, et al. Dietary glycemic load and cancer recurrence and survival in patients with stage III colon cancer: Findings from CALGB 89803. J Natl Cancer Inst. 2012;104(22):1702-1711.

11. Holt SH, Miller JC, Petocz P. An insulin index of foods: The insulin demand generated by 1000-kJ portions of common foods. Am J Clin Nutr. 1997;66(5): 1264-1276.

12. Gannon MC, Nuttall FQ, Neil BJ, et al. The insulin and glucose responses to meals of glucose plus various proteins in type II diabetic subjects. Metabolism. 1988;37(11):1081-1088.

13. Gannon MC, Nuttall FQ, Westphal SA, et al. The effect of fat and carbohydrate on plasma glucose, insulin, C-peptide, and triglycerides in normal male subjects. J Am Coll Nutr. 1993;12(1):36-41.

14. Saltz LB, Niedzwiecki D, Hollis D, et al. Irinotecan fluorouracil plus leucovorin is not superior to fluorouracil plus leucovorin alone as adjuvant treatment for stage III colon cancer: Results of CALGB 89803. J Clin Oncol. 2007;25(23): 3456-3461.

15. Willett WC, Sampson L, Stampfer MJ, et al. Reproducibility and validity of a semiquantitative food frequency questionnaire. Am J Epidemiol. 1985;122(1): $51-65$.

16. Willett WC, Reynolds RD, Cottrell-Hoehner S, et al. Validation of a semiquantitative food frequency questionnaire: Comparison with a 1-year diet record. J Am Diet Assoc. 1987;87(1):43-47.

17. Willett W, Stampfer MJ. Total energy intake: Implications for epidemiologic analyses. Am J Epidemiol. 1986;124(1):17-27.

18. Bao J, Atkinson F, Petocz P, et al. Prediction of postprandial glycemia and insulinemia in lean, young, healthy adults: Glycemic load compared with carbohydrate content alone. Am J Clin Nutr. 2011;93(5):984-996.

19. Hu FB, Stampfer MJ, Rimm E, et al. Dietary fat and coronary heart disease: A comparison of approaches for adjusting for total energy intake and modeling repeated dietary measurements. Am J Epidemiol. 1999;149(6):531-540.

20. Bertagnolli MM, Niedzwiecki D, Compton CC, et al. Microsatellite instability predicts improved response to adjuvant therapy with irinotecan, fluorouracil, and leucovorin in stage III colon cancer: Cancer and Leukemia Group B Protocol 89803. J Clin Oncol. 2009;27(11):1814-1821.

21. Ogino S, Kawasaki T, Brahmandam M, et al. Sensitive sequencing method for KRAS mutation detection by pyrosequencing. J Mol Diagn. 2005;7(3):413-421.

22. Ogino S, Meyerhardt JA, Irahara N, et al. KRAS mutation in stage III colon cancer and clinical outcome following intergroup trial CALGB 89803. Clin Cancer Res. 2009;15(23):7322-7329.

23. Ogino S, Shima K, Meyerhardt JA, et al. Predictive and prognostic roles of BRAF mutation in stage III colon cancer: Results from intergroup trial CALGB 89803. Clin Cancer Res. 2012;18(3):890-900. 
24. Ogino S, Liao X, Imamura $\mathrm{Y}$, et al. Predictive and prognostic analysis of PIK3CA mutation in stage III colon cancer intergroup trial. J Natl Cancer Inst. 2013;105(23):1789-1798.

25. Warren RS, Atreya CE, Niedzwiecki D, et al. Association of TP53 mutational status and gender with survival after adjuvant treatment for stage III colon cancer: Results of CALGB 89803. Clin Cancer Res. 2013;19(20):5777-5787.

26.Therneau T, Grambsch P. Modeling Survival Data: Extending the Cox Model. New York: Springer; 2000.

27. Jones MP, Crowley J. A general class of nonparametric tests for survival analysis. Biometrics. 1989;45(1):157-170.

28. Kleinbaum DG, Klein M. Survival Analysis: A Self-Learning Text. 2nd ed. New York: Springer; 2005.

29. Reaven G. The metabolic syndrome or the insulin resistance syndrome? Different names, different concepts, and different goals. Endocrinol Metab Clin North Am. 2004;33(2):283-303.

30. Wilcox G. Insulin and insulin resistance. Clin Biochem Rev. 2005;26(2):19-39.

31. Yuan C, Bao Y, Sato K, et al. Influence of dietary insulin scores on survival in colorectal cancer patients. Br J Cancer. 2017;117(7):1079-1087.
32. Mardilovich K, Pankratz SL, Shaw LM. Expression and function of the insulin receptor substrate proteins in cancer. Cell Commun Signal. 2009;7:14.

33. Koda M, Reszec J, Sulkowska M, et al. Expression of the insulin-like growth factor-I receptor and proapoptotic Bax and Bak proteins in human colorectal cancer. Ann N Y Acad Sci. 2004;1030:377-383.

34. Giovannucci E, Harlan DM, Archer MC, et al. Diabetes and cancer: A consensus report. CA Cancer J Clin. 2010;60(4):207-221.

35. Meyerhardt JA, Heseltine D, Campos $\mathrm{H}$, et al. Assessment of a dietary questionnaire in cancer patients receiving cytotoxic chemotherapy. J Clin Oncol. 2005;23(33):8453-8460.

36. Hu FB, Stampfer MJ, Colditz GA, et al. Physical activity and risk of stroke in women. JAMA. 2000;283(22):2961-2967.

37. Hu FB, Stampfer MJ, Solomon C, et al. Physical activity and risk for cardiovascular events in diabetic women. Ann Intern Med. 2001;134(2):96-105.

38. Rockhill B, Willett WC, Manson JE, et al. Physical activity and mortality: A prospective study among women. Am J Public Health. 2001;91(4):578-583.

39. Hu FB, Willett WC, Li T, et al. Adiposity as compared with physical activity in predicting mortality among women. N Engl J Med. 2004;351(26):2694-2703. 